\title{
Review
}

\section{Column Chromatography}

Proceedings of the 5th International Symposium on Separation Methods held in Lausanne (Switzerland), October 7-10, 1969. E. sz. Kovats Ed., Sauerländer, Aarau (Switzerland), 1970.

This meeting, organized in Lausanne by $E$. Kovats in October 1969, was the 5th International Symposium in the series. It was also probably the most successful one, due to the care with which Dr. Kovats chose the papers and to his exquisite sense of hospitality.

The proceedings of this meeting were published a few months ago. Thus the many interesting papers delivered there are now generally available. Most fields of chromatography have been discussed and each of the nine sections of the book contains papers on theoretical or applied problems which are worth reading. These sections are:

1. Electrophoresis and chromatography

2. Liquid column chromatography

3. Engineering treatment of chromatography

4. Reaction chromatography

5. Gel permeation chromatography

6. Chromatographic retention data

7. Supercritical fluid chromatography

8. Chromatography of labile and corrosive substances

9. Other topics.

The topic of each half-day session was determined by the plenary lecture, which was followed by communications on the same topic and by extensive and fruitful discussions. The papers are similarly aranged in the book.
One of the main sections is devoted to the new, fastexpanding field of liquid chromatography, and contains an important lecture by Dr. Huber and some very interesting communications. Among the other highlights of the meeting were the first plenary lecture by $\mathrm{Dr}$. A. J. P. Martin who presented his recent work on displacement electrophoresis, a new, powerful method of separation which might have a bright future in the field of protein fractionation; the lively discussion on the standardization of liquid phases, excellently reported by Dr. Kovats; and the lecture by Dr. Villermaux on a new chemical engineering approach to the theory of chromatography. Other interesting contributions were an important paper by Pr. Kiselev on adsorption from multicomponent solutions and an interesting review by $D r$. Beroza on the coupling of chemical reactions, and chromatography for the purpose of identification. Many other communications are worth quoting but might well turn this review into a comment on the table of contents.

It should be underlined, however, that analysts concerned with practical problems will find important information in the sections on retention data and on chromatography of labile and corrosive substances.

This book is well presented. Most of the papers are in English ( 27 communications out of 44,4 plenary lectures out of 9). The others are in German $(12+3)$, or in French $(5+2)$. The lack of an English summary and of an English translation of the figure captions for the papers written in French and German is the only notable defect of a very useful book which has a place on most chromatographers' bookshelves.

G. Guiochon 